\title{
ROLE OF THYROID BIOMARKERS IN ASSOCIATION WITH SOFA SCORE IN PREDICTING THE PROGNOSIS OF POST-OPERATIVE CRITICALLY ILL PATIENTS
}

\author{
Pallavi Rain1, Maliyannar Itagappa², Satish Kumar ${ }^{3}$, Shaila Mitra4, Ashish Agrawal ${ }^{5}$
}

1Research Scholar, Department of Biochemistry, Santosh University, Ghaziabad, Uttar Pradesh, India.

2Professor, Department of Biochemistry, Santosh University, Ghaziabad, Uttar Pradesh, India.

3 Professor, Department of Anaesthesia and Critical Care Medicine, Baba Raghav Das Medical College, Gorakhpur, Uttar Pradesh, India.

4 Professor, Department of Pathology, Baba Raghav Das Medical College, Gorakhpur, Uttar Pradesh, India.

${ }_{5}^{5}$ Senior Resident, Department of Anaesthesia and Critical Care Medicine, Baba Raghav Das Medical College, Gorakhpur, Uttar Pradesh, India.

\section{BACKGROUND}

\section{ABSTRACT}

Thyroid hormone alterations in critically ill patients are very common and may add to the severity of disease and the outcome s of patients in Intensive Care Unit (ICU). This observational study was conducted to detect the thyroid biomarkers as independent predictors of ICU mortality to predict prognosis more accurately in post-operative critically ill ICU patients with sepsis.

This study also tries to determine any association between the thyroid biomarkers and SOFA (sepsis-related organ failure assessment) score.

\section{MATERIALS AND METHODS}

This is a prospective observational study. We investigated free triiodothyronine (FT3), free thyroxine (FT4) and Thyroid Stimulating Hormone (TSH) in low SOFA score patients (LSS; $n=21$, SOFA $=0-6$ ) and in high SOFA score patients (HSS; $n=82$, SOFA > 6). The serum levels of FT3, FT4 and TSH were correlated with SOFA score. Further, prognosis on fifth day of ICU admission was defined on the basis of SOFA score, FT3, FT4 and TSH.

\section{RESULTS}

The serum levels of FT3 $(p=0.004)$, FT4 $(p=0.025)$ and TSH $(p>0.05)$ decreased in HSS than LSS. A negative correlation was observed between thyroid biomarkers and SOFA score $(>6)$. Patients who deteriorated on the fifth day of ICU admission had significantly high SOFA score $(\mathrm{p}=2.15 \mathrm{E}-06)$ and significantly low FT3 $(\mathrm{p}=3.17 \mathrm{E}-10)$, FT4 $(\mathrm{p}=4.06 \mathrm{E}-05)$ and TSH $(\mathrm{p}=2.21 \mathrm{E}-05)$ on first day of ICU admission than those who improved on fifth day.

\section{CONCLUSION}

Thyroid biomarkers, specifically low FT3 might be an efficient biomarker or risk factor in evaluating prognosis of the postoperative critically ill patients.

\section{KEY WORDS}

Critically Ill Patients, Sepsis, Thyroid Hormone, Triiodothyronine, Thyroxine, Thyroid Stimulating Hormone, SOFA Score.

HOW TO CITE THIS ARTICLE: Rain P, Itagappa M, Kumar S, et al. Role of thyroid biomarkers in association with SOFA score in predicting the prognosis of post-operative critically ill patients. J. Evolution Med. Dent. Sci. 2018;7(42):4550-4555, DOI: $10.14260 /$ jemds/2018/1015

\section{BACKGROUND}

Critical illness with sepsis exerts a state of stress which results in hypermetabolism, increased energy expenditure, hyperglycaemia and muscle loss. Thus, the metabolic response to sepsis involves every organ and tissue of the body and yet very little is known about the underlying mechanism. In critically ill patients, thyroid hormone dysfunction is noted even when there is no history of previous intrinsic thyroid disease, ${ }^{1-3}$ often referred as Euthyroid Sick Syndrome (ESS) or Non-Thyroidal Illness Syndrome (NTIS). ${ }^{4}$

'Financial or Other Competing Interest': None.

Submission 05-09-2018, Peer Review 29-09-2018,

Acceptance 05-10-2018, Published 15-10-2018.

Corresponding Author:

Pallavi Rain,

Research Scholar, Department of Biochemistry,

Santosh Medical College, Santosh University,

No. 1, Santosh Nagar, Ghaziabad-201009,

Uttar Pradesh, India.

E-mail: pallavirain18@gmail.com

DOI: $10.14260 /$ jemds/2018/1015

\section{(c) $(1) \ominus$}

ESS is characterised by low level of total triiodothyronine (T3) and free T3 (FT3) and high level of reverse T3 (rT3) along with normal or low level of thyroxine (T4) and thyroid stimulating hormone (TSH).5,6 Thyroid hormones play an important role in the adaptation of metabolic function to stress and critical illness. ${ }^{7}$

Thyroid hormone alterations, particularly in old patients or in those with critical illness are very common and are correlated with the severity of disease and the outcomes of patient in Intensive Care Unit (ICU). ${ }^{8-11}$ Low T3 is commonly observed in critically ill patients, which is attributed to increased deiodination of T4 to rT3 rather than to T3 and increased catabolism of $\mathrm{T} 3$ to 3, 3-diiodothyronine $\left(\mathrm{T}_{2}\right)$. $^{12-14}$ Low total T4 and free T4 (FT4) and sometimes low TSH may be observed with increasing severity of illness. ${ }^{14}$ Alteration in thyroid hormone levels during critical illness may also be attributed to decrease in plasma $\mathrm{T}_{4}$-binding globulin or transthyretin and accumulation of substances that lower the plasma thyroid hormone-binding capacity. 12 Studies have mentioned that low thyroid hormone are independent predictors of mortality in patients admitted to ICU like other 
parameters such as hyperglycaemia, hyperprolactinaemia, procalcitonin (PCT), brain natriuretic peptide (BNP) etc. suggesting the inclusion of the thyroid profile in the scoring system, which are used to predict the mortality of the ICU patients. ${ }^{15-17}$

Sequential organ failure assessment or sepsis-related organ failure assessment score (SOFA score) is used to predict the severity of the critically ill patients admitted in ICU. SOFA score was developed to quantify the severity of patient's illness based on the degree of organ dysfunction data on six organ failures, i.e. haematologic-platelets count, hepatic-serum bilirubin, renal-serum creatinine, cardiovascular- mean arterial pressure (MAP), central nervous system (CNS)- Glasgow coma score (GCS), respiratory-PaO2/FIO2. Although, there is no direct conversion of SOFA score to mortality, a rough estimate of mortality risk may be made. ${ }^{18-20}$ The mortality risk is $>25 \%$ if SOFA score is $>9$ and $>50 \%$ if SOFA score is $>13.20$

Sequential assessment of organ dysfunction during the first few days of ICU admission is a good indicator of prognosis. Both the mean and highest SOFA scores are particularly useful predictors of outcome. Regardless of the initial score, an increase in SOFA score during the first $48 \mathrm{~h}$ in the ICU predicts a mortality rate of at least $50 \% .21,22$ Vosylius et al showed that cumulative SOFA scores were better in discriminating the outcome compared to a single organ dysfunction scores. ${ }^{23}$

Low level of thyroid hormone is correlated with severity of illness along with SOFA in various studies. ${ }^{24-29}$ Therefore, this observational study was intended to detect the thyroid biomarkers (FT3, FT4 and TSH) as independent predictors of ICU mortality in unselected post-operative patients. This study may be helpful in adding one more parameter to define the prognosis of these critically ill post-operative patients with sepsis along with SOFA, APACHE II and others.

\section{MATERIALS AND METHODS \\ Ethics}

The prospective observational study design was approved by the Ethics Review Committee of Santosh University, Ghaziabad, Uttar Pradesh and Baba Raghav Das (BRD) Medical College, Gorakhpur, Uttar Pradesh. All procedures were performed in accordance to the tenets of Helsinki Declaration. The study was explained to each subject and informed consent was obtained from each participant.

\section{Study Samples}

The prospective observational study design was conducted on post-surgery critically ill patients admitted in the ICU under Department of Anaesthesia at BRD Medical College, Gorakhpur. A total of 103 post-operative critically ill subjects with sepsis were recruited in the study. Sepsis was defined by the presence of any two conditions among 1) Temperature $>38^{\circ} \mathrm{C}$ or $<36^{\circ} \mathrm{C}, 2$ ) Heart rate $>90$ beats $/ \mathrm{min}, 3$ ) White blood cell count $>12,000$ or $<4,000$ cells $/ \mathrm{mm}^{2}$ and 4) Respiratory rate $>20$ breaths $/ \mathrm{min}$ or $\mathrm{PaCO}_{2}<32 \mathrm{mmHg}$. SOFA score was recorded and sample was collected on the first day of admission to ICU. Prognosis of the patients was recorded on the $5^{\text {th }}$ day of ICU admission.

\section{Exclusion Criteria}

Young patients $(<18$ years) were not included in the study. Subjects were excluded from the study if they were under any hormonal therapy or taking amiodarone or had a previous history of thyroid diseases such as hyperthyroidism, hypothyroidism and thyroid tumours. Patients were also excluded if thyroid nodule was observed by physical examination when admitted to the ICU. Patients were excluded if they died or were discharged from the ICU within 4 hours of admission. Pregnant women and women receiving oral contraceptive or hormonal replacement therapy were excluded from the study. Participants were not recruited if there was inability to give consent or do not comply with the study protocol.

\section{Limitations}

Since the calculated sample size was too high and thereby not feasible to include in this limited period of study, we had to limit the sample size for convenience.

\section{Sample Collection}

Five $\mathrm{mL}$ of blood sample was drawn in the vacutainer on first day of ICU admission. The blood was clotted and centrifuged (Remi Laboratory Instruments, Mumbai, Maharashtra, India) for 10 minutes at $1500 \mathrm{rpm}$ to collect serum for estimation of biomarkers.

\section{Biomarker Estimation}

ARCHITECT free T3, ARCHITECT free T4 and ARCHITECT TSH (ARCHITECT System, Abbott Diagnostics, Illinois, USA) were used for the quantitative determination of FT3, FT4 and TSH, respectively, in serum. These kits are based on chemiluminescent microparticle immunoassay.

\section{Biostatistical Analyses}

Data analysed by using GraphPad Prism version 5, P-value $\leq 0.05$ was considered for statistical significance. P-value $\leq 0.001$ was considered for high statistical significance. Tests:Fisher's Exact test, unpaired t-test, SPSS 16.0 (SPSS Inc., Chicago, Illinois, USA) and Epi Info ${ }^{\mathrm{TM}} 6.0$ (Centres for Disease Control, Atlanta, Georgia, USA) software were used for statistical analysis. Statistical power for biomarker study was calculated by Simple Interactive Statistical Analysis (SISA) online

tool (http://www.quantitativeskills.com/sisa/calculations/power .htm). The power for all the significant biomarkers was $\geq 95 \%$. Further, the biochemical parameters were expressed as mean \pm SE (Standard Error), whereas the significance in levels were analysed using SPSS 16.0. A p-value of $\leq 0.05$ was considered as statistically significant.

\section{RESULTS}

\section{Clinical Characteristics}

Table 1 highlights the clinical characteristics of the two study groups, i.e. LSS and HSS. Age and gender differed among the two groups. Hence, the biochemical data was adjusted with these factors as confounding. The average age of LSS was $27.71 \pm 9.69$ and for HSS $40.21 \pm 17.3(p=0.041)$. In LSS 9.5\% patients were male and $90.5 \%$ were female, while in HSS $42.7 \%$ patients were male and $57.3 \%$ were female $(p=0.005)$. 
The average SOFA score in LSS was $5.9 \pm 0.44$ and it was $10.51 \pm 2.48$ in $\operatorname{HSS}(\mathrm{p}=0.0001$; Table 1$)$.

The Study Samples were divided into Two Groups based on the SOFA Score as following

1. Group 1 or low SOFA score group (LSS; $n=21$, where ' $n$ ' is number of subjects): Patients with SOFA score 0 - 6; this was the reference group of our study.

2. Group 2 or high SOFA score group (HSS; $n=82$ ): Patients with SOFA score $\geq 7$.

\section{Biomarker Serum Levels}

Serum level of FT3 at $1.73 \pm 0.31 \mathrm{pg} / \mathrm{mL}$ was reduced significantly in HSS than the level $2.03 \pm 0.22 \mathrm{pg} / \mathrm{mL}$ in LSS $(p=0.004$; Figure 1a). Similarly, serum level of FT4 at $0.94 \pm 0.23 \mathrm{ng} / \mathrm{dL}$ was reduced significantly in HSS than the level $1.14 \pm 0.20 \mathrm{ng} / \mathrm{dL}$ in LSS ( $\mathrm{p}=0.025$; Figure $1 \mathrm{~b}$ ). Further, serum level of TSH at $1.84 \pm 1.16 \mu \mathrm{IU} / \mathrm{mL}$ was reduced in HSS than the level $2.54 \pm 0.54 \mu \mathrm{IU} / \mathrm{mL}$ in LSS $(\mathrm{p}=0.167$; Figure 1c).

\section{Biomarker Correlations}

The serum levels of FT3, FT4 and TSH were correlated with SOFA score. The serum levels of FT3, FT4 and TSH decreased with increase in SOFA score. Remarkable decrease in FT3 was observed after 12 SOFA scores, while in FT4 and TSH a remarkable decrease was observed after 11 SOFA scores (Figure 2a, 2b and 2c).

Further, the HSS group was divided into three subgroups based on the prognosis on the fifth day of ICU admission. Patients in HSS group either showed no difference in their condition as on the first day of admission or they showed improved or deteriorated condition. We compared the three prognosis types with each other in terms of SOFA score, FT3, FT4 and TSH (Figure 3).

We observed that the patients who improved had significantly lower SOFA score than the patients with static or deteriorated condition. There was no difference between patients with static and deteriorated condition with regard to SOFA score (Figure 3a). Interestingly, we found that the improved patients had significantly higher serum level of FT3, FT4 and TSH than both, patients with static and deteriorated condition (Figure 3b, 3c and 3d). The FT3, FT4 and TSH serum levels followed a decreasing trend with highest levels in improved patients followed by patients with static condition and lowest in deteriorated patients.

\begin{tabular}{|c|c|c|c|}
\hline $\begin{array}{c}\text { Clinical } \\
\text { Characteristics }\end{array}$ & LSS (n=21) & HSS (n=82) & P-value \\
\hline Age (Years) & $27.71 \pm 9.69$ & $40.26 \pm 17.26$ & 0.040 \\
\hline Gender & $\begin{array}{c}\mathrm{M}=2(9.5 \%) \\
\mathrm{F}=19(90.5 \%)\end{array}$ & $\begin{array}{c}\mathrm{M}=35(42.7 \%) \\
\mathrm{F}=47(57.3 \%)\end{array}$ & 0.005 \\
\hline SOFA Score & $5.90 \pm 0.44$ & $10.51 \pm 2.48$ & 0.0001 \\
\hline \multicolumn{4}{|c|}{ Table 1. Clinical Characteristics of the Two Groups } \\
\hline
\end{tabular}

The data are presented as mean \pm standard deviation except gender, which is represented as number of samples (percentage). P-values were calculated using the Epi Info $^{\mathrm{TM}}$ 6.0. A p-value of $\leq 0.05$ was considered significant. Representations: n, Number of samples; M, Male; F, Female; SOFA score, Sepsis-related organ failure assessment score.
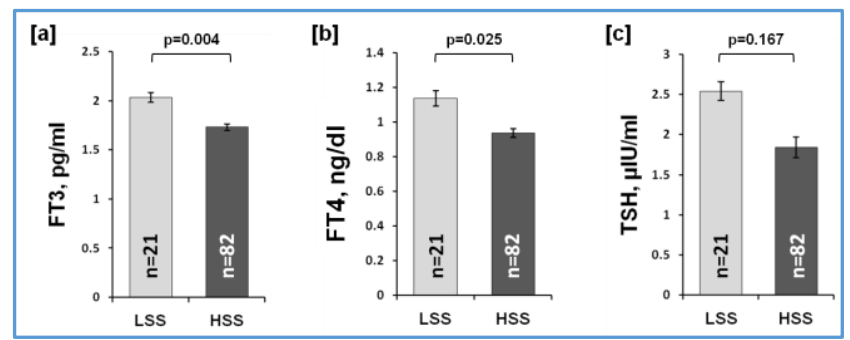

Figure 1. Serum Levels of (a) FT3 (b) FT4 and (c) TSH in LSS and HSS

The data are presented as mean \pm SE. SPSS 16.0 was used to perform GLM and obtain p-value after adjustment with age and gender. A p-value of $\leq 0.05$ was considered significant. Representations: FT3, free triiodothyronine; FT4, free thyroxine; TSH, thyroid stimulating hormone; LSS, low SOFA score group; HSS, high SOFA score group; $p$, pvalue; $n$, Number of samples.

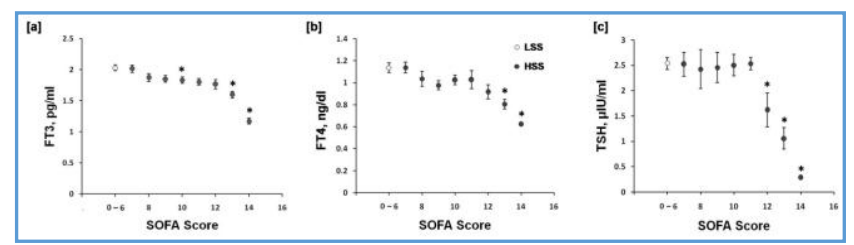

Figure 2. Correlation between SOFA Score and (a) FT3 (b) FT4 and (c) TSH in LSS and HSS

The data are presented as mean \pm SE. SPSS 16.0 was used to perform GLM and obtain p-value after adjustment with age and gender. A p-value of $\leq 0.05$ was considered significant, $* \mathrm{P} \leq 0.05$ when compared to LSS i.e. patients with 0-6 SOFA score. Representations: FT3, free triiodothyronine; FT4, free thyroxine; TSH, thyroid stimulating hormone; SOFA score, sepsis-related organ failure assessment score; LSS, low SOFA score group; HSS, high SOFA score group.

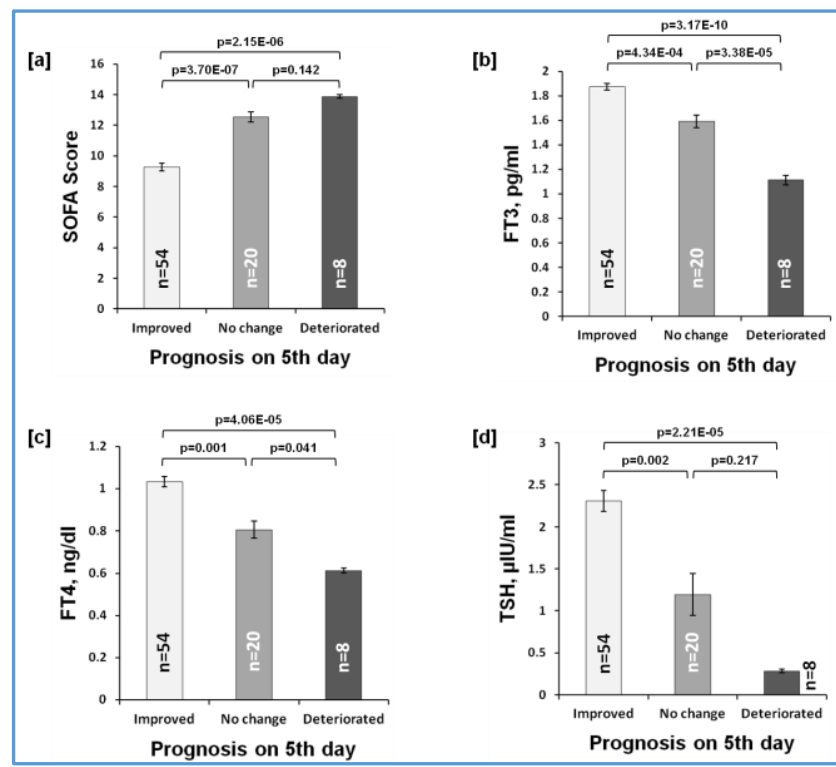

Figure 3. Trend of (a) SOFA Score, (b) FT3, (c) FT4 and (d) TSH among Patients with different Prognosis

The data are presented as mean \pm SE. SPSS 16.0 was used to perform GLM and obtain p-value after adjustment with age and gender. A p-value of $\leq 0.05$ was considered significant. 
Representations: SOFA score, sepsis-related organ failure assessment score; FT3, free triiodothyronine; FT4, free thyroxine; TSH, thyroid stimulating hormone; LSS, low SOFA score group; HSS, high SOFA score group; $\mathrm{p}$, p-value; $\mathrm{n}$, number of samples.

\section{DISCUSSION}

Thyroid dysfunction in critically ill patients is the most common observation. However, whether thyroid dysfunction can be considered as a risk factor to predict patient's outcome is still debatable. Various studies have reported that nonsurvivors had very low thyroid biomarkers as compared to the survivors. ${ }^{27-29}$ Although, most of the studies suggest definite association between thyroid dysfunction and worse outcome in sepsis, the findings are inconsistent and need further investigation. 30

In our study we observed the average serum FT3 level $1.73 \pm 0.31 \mathrm{pg} / \mathrm{mL}$ in HSS group, while it was $2.03 \pm 0.22$ $\mathrm{pg} / \mathrm{mL}$ in LSS group, though both the reading are in normal range; however, in HSS group it was significantly on lower side $(\mathrm{p}=0.004$; Figure 1a). About 22 patients $(26.8 \%$ patients), in total 82 subjects in HSS group were having FT3 value below normal. FT4 was also significantly on lower side in HSS group, $0.94 \pm 0.23 \mathrm{ng} / \mathrm{dL}$ as compared to LSS group where the average FT4 was $1.14 \pm 0.20 \mathrm{ng} / \mathrm{dL}(\mathrm{p}=0.025$; Figure $1 \mathrm{~b})$. However, 17 patients (20.7\% patients) in HSS group showed FT4 level below normal. Further, the average TSH was $2.54 \pm 0.54 \mu \mathrm{IU} / \mathrm{mL}$ in LSS group and $1.84 \pm 1.16$ $\mu \mathrm{IU} / \mathrm{mL}$ in HSS group, though this change is insignificant $(p=0.167$; Figure 1c) but the value in HSS group is on lower side. Among HSS, 13 patients $(15.85 \%)$ even have got the value below the lower limit of normal range i.e. $0.35 \mu \mathrm{IU} / \mathrm{mL}$.

The changes in thyroid hormone economy in post-surgical critically ill patients observed may be because of peripheral metabolism of the thyroid hormone, alterations in TSH regulation and alteration in binding of thyroid hormone to thyroid binding globulin. Acute inhibition of type-I 5'deiodinase also play an important role in decreasing level of T3 in such patients. ${ }^{31-33}$ Role of some cytokines, notably tumour necrosis factor- $\alpha$ (TNF- $\alpha$ ), interleukin 1 (IL-1) and IL6 has also been observed by researchers. ${ }^{34}$ Therefore, thyroid dysfunction may be a general finding in critically ill patients irrespective of aetiology of the illness (medical or postsurgical).

When these biomarkers are correlated with SOFA score, it was found that FT3, FT4 and TSH start decreasing when SOFA score is $>11$. However, FT3 decreases more remarkably when SOFA score is more than 12 (Figure 2a, 2b and 2c). So, it seems that there is definite negative correlation between thyroid hormones and SOFA score. Hosny et al and Türe et al also observed decreasing FT3 with increasing SOFA score in septic and Acute Respiratory Distress Syndrome (ARDS) patients, respectively. ${ }^{27,28}$ FT3 levels might have additive discriminatory power to age, SOFA and APACHE II scores in predicting short-term mortality in ARDS patients. ${ }^{28}$

Further, we observed the prognosis of the patients on fifth day. All the patients in LSS group improved. In HSS group, we noticed the patients who improved $(n=54)$ has less SOFA score as compared to the patients in which there was clinically no change $(n=20)$. The patients who further deteriorated $(n=8)$ had even higher SOFA score (Figure 3a). The FT3 was well within normal range in the patients who improved, and it is below normal in the patients who deteriorated (Figure $3 \mathrm{~b}$ ). The FT4 value was again below normal range in patients who deteriorated as compared to the patients who showed no sign of improvement, where FT4 value was slightly on higher side and the value was well within normal range in improved patients (Figure 3c). In the deteriorated patients, the TSH value was on lower side or below normal range. While in other patients whether they improved or in status quo had their TSH value within normal range (Figure $3 \mathrm{~d}$ ). So, the observations suggest that patients who had lower thyroid biomarkers value might deteriorate further with the same treatment protocol.

Low serum T3 levels have also been observed in muscle and liver during critical illness leading to higher mortality. ${ }^{35}$ Thyroid treatment might be helpful in improving the condition of the critically ill patients, but the studies have reported inconsistent finding. Young and Worthley in their clinical practical review noted abnormal thyroid function, which improves when patient's general condition improves, so generally does not require thyroid supplementation. ${ }^{36}$ Further, Kumar et al observed marked suppression of FT3 with normal TSH in critically ill patients and they noticed improvement in their patients with intravenous levothyroxine treatment. ${ }^{37}$

In post-operative surgical patients thyroid dysfunction is often observed, especially when associated with septicaemia. ${ }^{38}$ Hence, it is obligatory to investigate that thyroid dysfunction might be a risk factor and whether controlling it would decrease the mortality rate in surgically ill patients. Bunevicius et al have shown that low T3 syndrome is a strong independent predictor of unfavourable clinical outcome and depressive symptoms in patients that went under brain tumour surgery. 39 Therefore, the free T3 concentration might be considered as a prognostic biomarker in neurosurgical critically ill patients. To generalise the above finding, we performed our study in post-operative surgical patients including the post-operative neurosurgical patients. We observed that thyroid dysfunction, especially low FT3 levels can predict worse outcome in post-operative surgical patients with high SOFA score on the first day of admission to ICU. However, further study is required in a larger sample size and including patients with higher SOFA score than our study. Increasing the observation timeline to more than 5 days, i.e. until recovery or demise and determining thyroid biomarkers on successive days will further increase the significance of study.

\section{Limitations}

Since the calculated sample size was too high and thereby not feasible to include in this limited period of study, we had to limit the sample size for convenience.

\section{CONCLUSION}

After careful observations of our results, we concluded that there is definite correlation between thyroid biomarkers and SOFA score. Thyroid profile decreases with increase in SOFA score. These biomarkers, specifically FT3 might act as an effective tool in evaluating prognosis of the post-operative critically ill patients. However, more studies with large sample size should be done to establish this finding in clinical use. 


\section{ACKNOWLEDGEMENTS}

We acknowledge the technical support of the staff of central lab and ICU at BRD Medical College, Gorakhpur, Uttar Pradesh. We are thankful to Manjari Rain for helping us with the analyses. We thank all the volunteers who participated in the study.

\section{REFERENCES}

[1] Farwell AP. Sick euthyroid syndrome in the intensive care unit. In: Irwin RS, Rippe JM, eds. Irwin and Rippe's Intensive Care Medicine. Philadelphia: Lippincott Williams \& Wilkins 2003: p. 1205-16.

[2] DeGroot LJ. "Non-thyroidal illness syndrome" is functional central hypothyroidism and if severe, hormone replacement is appropriate in light of present knowledge. J Endocrinol Invest 2003;26(12):1163-70.

[3] Adler SM, Wartofsky L. The non-thyroidal illness syndrome. Endocrinol Metab Clin North Am 2007;36(3):657-72, vi.

[4] Sahana PK, Ghosh A, Mukhopadhyay P, et al. A study on endocrine changes in patients in intensive care unit. J Indian Med Assoc 2008;106(6):362-4.

[5] Docter R, Krenning EP, de Jong M, et al. The sick euthyroid syndrome: changes in thyroid hormone serum parameters and hormone metabolism. Clin Endocrinol (Oxf) 1993;39(5):499-518.

[6] Mclver B, Gorman CA. Euthyroid sick syndrome: an overview. Thyroid 1997;7(1):125-32.

[7] Lodha R, Vivekanandhan S, Sarthi M, et al. Thyroid function in children with sepsis and septic shock. Acta Paediatr 2007;96(3):406-9.

[8] Iglesias P, Muñoz A, Prado F, et al. Alterations in thyroid function tests in aged hospitalized patients: prevalence, aetiology and clinical outcome. Clin Endocrinol (Oxf) 2009;70(6):961-7.

[9] Simons RJ, Simon JM, Demers LM, et al. Thyroid dysfunction in elderly hospitalized patients. Effect of age and severity of illness. Arch Intern Med 1990;150(6):1249-53.

[10] Marx C, Petros S, Bornstein SR, et al. Adrenocortical hormones in survivors and nonsurvivors of severe sepsis: diverse time course of dehydroepiandrosterone, dehydroepiandrosteronesulfate and cortisol. Crit Care Med 2003;31(5):1382-8.

[11] Schuetz P, Müller B, Nusbaumer C, et al. Circulating levels of $\mathrm{GH}$ predict mortality and complement prognostic scores in critically ill medical patients. Eur J Endocrinal 2009;160(2):157-63.

[12] Warner MH, Beckett GJ. Mechanisms behind the nonthyroidal illness syndrome: an update. J Endocrinol 2010;205(1):1-13.

[13] Sakharova OV, Inzucchi SE. Endocrine assessments during critical illness. Crit Care Clin 2007;23(3):46790.

[14] Peeters RP, Wouters PJ, Kaptein E, et al. Reduced activation and increased inactivation of thyroid hormone in tissues of critically ill patients. J Clin Endocrinol Metab 2003;88(7):3202-11.

[15] Peeters RP, Wouters PJ, van Toor H, et al. Serum 3, 3', $5^{\prime}$-triiodothyronine (rT3) and 3,5,3'triiodothyronine/rT3 are prognostic markers in critically ill patients and are associated with postmortem tissue deiodinase activities. J Clin Endocrinol Metab 2005;90(8):4559-65.

[16] Slag MF, Morley JE, Elson MK, et al. Hypothyroxinemia in critically ill patients as a predictor of high mortality. JAMA 1981;245(1):43-5.

[17] Lim DJ, Herring MK, Leef KH, et al. Hypothyroxinemia in mechanically ventilated term infants is associated with increased use of rescue therapies. Pediatrics 2005;115(2):406-10.

[18] Vincent JL, Moreno R, Takala J, et al. The SOFA (Sepsisrelated Organ Failure Assessment) score to describe organ dysfunction/failure. On behalf of the Working Group on Sepsis-Related Problems of the European Society of Intensive Care Medicine. Intensive Care Med 1996;22(7):707-10.

[19] Vincent JL, de Mendonça A, Cantraine F, et al. Use of the SOFA score to assess the incidence of organ dysfunction/failure in intensive care units: results of a multicenter, prospective study. Working group on "sepsis-related problems" of the European Society of Intensive Care Medicine. Crit Care Med 1998;26(11):1793-800.

[20] Rapsang AG, Shyam DC. Scoring systems in the intensive care unit: a compendium. Indian J Crit Care Med 2014;18(4):220-8.

[21] Ferreira FL, Bota DP, Bross A, et al. Serial evaluation of the SOFA score to predict outcome in critically ill patients. JAMA 2001;286(14):1754-8.

[22] Bale C, Kakrani AL, Dabadghao VS, et al. Sequential organ failure assessment score as prognostic marker in critically ill patients in a tertiary care intensive care unit. International Journal of Medical and Public Health 2013;3(3):155-8.

[23] Vosylius S, Sipylaite J, Ivaskevicius J. Sequential organ failure assessment score as the determinant of outcome for patients with severe sepsis. Croat Med J 2004;45(6):715-20.

[24] Ray DC, Macduff A, Drummond GB, et al. Endocrine measurements in survivors and non-survivors from critical illness. Intensive Care Med 2002;28(9):1301-8.

[25] Chinga-Alayo E, Villena J, Evans AT, et al. Thyroid hormone levels improve the prediction of mortality among patients admitted to the intensive care unit. Intensive Care Med 2005;31(10):1356-61.

[26] Ray DC, Drummond GB, Wilkinson E, et al. Relationship of admission thyroid function tests to outcome in critical illness. Anaesthesia 1995;50(12):1022-5.

[27] Hosny M, Rashad R, Atef D, et al. Predictive value of thyroid hormone assessment in septic patients in comparison with C-reactive protein. The Egyptian Journal of Critical Care Medicine 2015;3(2-3):55-61.

[28] Türe M, Memiş D, Kurt I, et al. Predictive value of thyroid hormones on the first day in adult respiratory distress syndrome patients admitted to ICU: comparison with SOFA and APACHE II scores. Ann Saudi Med 2005;25(6):466-72.

[29] Kumar KV, Kapoor U, Kalia R, et al. Low triiodothyronine predicts mortality in critically ill patients. Indian J Endocrinol Metab 2013;17(2):285-8. 
[30] Angelousi AG, Karageorgopoulos DE, Kapaskelis AM, et al. Association between thyroid function tests at baseline and the outcome of patients with sepsis or septic shock: a systematic review. Eur J Endocrinol 2011;164(2):147-55.

[31] Chopra IJ, Hershman JH, Pardridge WM, et al. Thyroid function in non-thyroidal illness. Annals of Internal Medicine 1983;98(6):946-57.

[32] Kaptein EM. Thyroid hormone metabolism in illness. In: Hennemann G, edr. Thyroid hormone metabolism. New York: Marcel Dekker 1986: p. 297-333.

[33] Nicoloff JT, Lopresti JS. Nonthyroidal illness. In: Braverman LE, Utiger RD, eds. The thyroid. $7^{\text {th }}$ edn. Philadelphia: Lippincott-Raven 1996: p. 286-96.

[34] Farwell AP. Sick euthyroid syndrome. J Intensive Care Med 1997;12(5):249-60.
[35] Peeters RP, van der Geyten S, Wouters PJ, et al. Tissue thyroid hormone levels in critical illness. J Clin Endocrinol Metab 2005;90(12):6498-507.

[36] Young R, Worthley LI. Diagnosis and management of thyroid disease and the critically ill patient. Crit Care Resusc 2004;6(4):295-305.

[37] Kumar E, McCurdy MT, Koch CA, et al. Impairment of thyroid function in critically ill patients in the intensive care units. Am J Med Sci 2018;355(3):281-5.

[38] Zaloga GP, Chernow B, Smallridge RC, et al. A longitudinal evaluation of thyroid function in critically ill surgical patients. Ann Surg 1985;201(4):456-64.

[39] Bunevicius A, Deltuva V, Tamasauskas S, et al. Low triiodothyronine syndrome as a predictor of poor outcomes in patients undergoing brain tumor surgery: a pilot study: clinical article. J Neurosurg 2013;118 (6):1279-87. 